\title{
Perfil de características ideales del docente de postgrado de pediatría
}

\author{
GUILLERMO ZEPEDA F. ${ }^{1}$, PEDRO HERSKOVIC L. ${ }^{2}$ \\ 1. Pediatra Universidad de Chile. Magíster en Educación en Ciencias de La Salud. Facultad de Medicina, Universidad de Chile. \\ 2 Pediatra. Departamento de Educación en Ciencias de la Salud y Departamento de Pediatría y Cirugía Infantil Oriente, \\ Facultad de Medicina, Universidad de Chile.
}

\begin{abstract}
Profile perception of ideal characteristics in postgraduate professor of pediatrics

Introduction: The ideal characteristics of the Graduate Professor of Pediatrics at the Medical School of the Universidad de Chile are yet not defined. Objective: To determine these characteristics from members' perception. Methodology: Qualitative research. Focus groups for Professors, alumni and Pediatrics students and interviews to three authorities took place at the Campus Oriente. Once the profile was elaborated, Directors of Departments of Pediatrics of other Campuses were interviewed for validation. Results: A teaching profile was elaborated differentiating the role of the professional as a person (committed, responsible, honest, respectful, tolerant, friendly, empathetic, assertive, leader, role model and able to maintain good interpersonal relationships), as a teacher (trained in teaching, planner, evaluator, with protected time for teaching, with capacities for self-reflection and self-assessment, learning facilitator, motivator, facilitator of a climate that encourages learning, communicator, with vocation, challenging, accessible, knowledgeable and user of new technologies and with ability to review the teaching process) and as a clinician (expert in his discipline, updated, researcher, identified and committed to the institution, able to work in a team). Conclusions: An ideal profile of the Graduate Professor of Pediatrics was created.

(Key words: Medical education, graduate, residency, competition, professor, pediatrics).

Rev Chil Pediatr 2013; 84 (2): 152-159
\end{abstract}

\section{RESUMEN}

Introducción: Las características ideales del docente de postgrado de pediatría de la Facultad de Medicina de la Universidad de Chile no están definidas. Objetivo: Determinar estas características a partir de las percepciones de sus integrantes. Metodología: Investigación cualitativa. En el Campus Oriente se realizó grupos focales de docentes, egresados y alumnos de Programa de Título de Especialista en Pediatría y entrevistas en profundidad a tres autoridades. Con este perfil elaborado se entrevistó a los directores de Departamento de

Recibido el 24 de mayo de 2012, devuelto para corregir el 30 de julio de 2012, segunda versión el 16 de agosto de 2012 , aceptado para publicación el 05 de febrero de 2013.

Tesis de Magíster en Educación en Ciencias de la Salud de la Facultad de Medicina de la Universidad de Chile. Este trabajo cumple con los requisitos sobre consentimiento /asentimiento informado, comité de ética, financiamiento, estudios animales y sobre la ausencia de conflictos de intereses según corresponda.

Correspondencia a:

Guillermo Zepeda Flores

E-mail: gezepeda@med.uchile.cl 
Pediatría de los restantes Campus para su validación. Resultados: Se elaboró un perfil docente diferenciando su rol como persona (comprometido, responsable, honesto, respetuoso, tolerante, acogedor, empático, asertivo, líder, modelo a seguir y capaz de mantener buenas relaciones interpersonales), como profesor (formado en docencia, planificador, evaluador, con tiempo protegido para la docencia, con capacidades de autoevaluación y de autorreflexión, facilitador del aprendizaje, motivador, propiciador de un clima que facilite el aprendizaje, comunicador, con vocación, exigente, accesible, conocedor y utilizador de nuevas tecnologías y con capacidad de revisar el proceso docente) y como clínico (experto en su disciplina, actualizado, investigador, identificado y comprometido con la institución, capaz de trabajar en equipo). Conclusiones: Se construyó un perfil ideal del docente de postgrado de pediatría.

(Palabras clave: Educación médica, Egresado, Residencia, Competencia, Docente, Pediatría).

Rev Chil Pediatr 2013; 84 (2): 152-159

\section{Introducción}

Las competencias del docente de postgrado de pediatría no están suficientemente definidas en los documentos oficiales de la Universidad de Chile ${ }^{1,2}$.

Por esto, en este punto es preciso reflexionar sobre lo siguiente: ¿estamos los docentes de Postgrado de la Universidad de Chile preparados para asumir la formación de los futuros pediatras?, ¿qué se espera de nosotros?, ¿cuáles son las características deseables en nuestro rol de docentes?.

Ahora bien, existen diferentes perfiles docentes en la literatura internacional tanto para el profesor universitario ${ }^{3,4}$, profesor universitario médico ${ }^{5,6}$, docente médico de pregrado $^{7}$, docente médico de postgrado ${ }^{8}$.

En este punto, es importante destacar que el proceso educativo es una práctica social históricamente localizada y culturalmente enraizada que sólo puede valorarse racionalmente situándola en la forma de vida social de la que surgió ${ }^{9}$.

Debido a lo anterior, al no existir información oficial acerca de las características deseables del docente de postgrado de pediatría y al no ser practicable plantear perfiles docentes creados en otros sitios con culturas diferentes a la nuestra se crea la necesidad de conocer e interpretar los habitus docentes ${ }^{10}$ del docente de postgrado de pediatría de la Facultad de Medicina de la Universidad de Chile, siendo el objetivo de esta investigación describir y determinar las características ideales de este docente a partir de las percepciones de los integrantes de esta cultura organizacional y así contribuir a la creación de un perfil docente propio de nuestra cultura.

\section{Metodología}

La investigación propuesta se enmarca dentro de un paradigma interpretativo el cual propone que el fenómeno educativo es una compleja red social y cultural en la cual múltiples realidades se construyen y consensuan intersubjetivamente ${ }^{11}$. El procedimiento 1levado a cabo es inductivo y su orientación es holística $^{12}$.

Esta investigación se realizó en su primera etapa entre el $2^{\circ}$ semestre de 2009 y el $1^{\text {er }}$ semestre de 2010 en el Campus Oriente de la Facultad de Medicina de la Universidad de Chile.

El diseño metodológico contempla el uso de técnicas cualitativas ${ }^{13-18}$, utilizando dos instrumentos de recolección de información: grupos focales y entrevistas en profundidad.

La realización de grupos focales fue con docentes, alumnos del programa de título de especialista en pediatría (APTEP) y egresados y se ocupó el criterio de máxima variación, cada grupo fue de ocho personas. Las entrevistas en profundidad se realizaron a personas considerados claves de esta cultura organizacional a saber, la Directora actual y el ex Director del Departamento de Pediatría y Cirugía Infantil del Campus Oriente y el jefe del Servicio de Pediatría del Hospital Luis Calvo Mackenna (Profesor Asociado de la Universidad de Chile y antiguo Director de Departamento).

Con este perfil de características elaborado en el Campus Oriente se entrevistó en profundidad a los Directores de los 4 Campus restan- 
tes de la Facultad de Medicina durante el $2^{\text {do }}$ semestre de 2010 con el objetivo de obtener su percepción de que las características ideales encontradas en la Sede Oriente también serían válidas en la sede donde son la principal autoridad académica.

Se confeccionó el guión de los grupos focales y entrevistas mediante el uso de preguntas abiertas utilizando las dimensiones de características explicitadas en la literatura ${ }^{19}$.

Las entrevistas tanto de los grupos focales como las entrevistas en profundidad fueron grabadas y transcritas. El procedimiento para verificar la concordancia entre la audición y la transcripción de lo grabado se realizó a saber, la transcripción de la grabación fue realizada por una persona contratada para ello y el autor del trabajo escuchó cada audición y simultáneamente chequeó detalladamente que hubiese sido una transcripción fiel. La duración de las grabaciones fue de 60 a $90 \mathrm{~min}$.

El análisis de los resultados se basó en el análisis de contenido ${ }^{12}$. Se realizó cada análisis de las entrevistas y de los grupos focales por separado y finalmente se sintetizó en la categorización y subcategorización finales. Cada uno de estos pasos fue realizado por separado por una psicóloga experta en investigación cualitativa y por el autor de esta investigación y el consenso logrado entre ambas partes luego de exponer cada cual su posición y establecer puntos de acuerdo fue lo que finalmente se expuso en el trabajo.

Nuestra investigación fue aprobada por el Comité de ética del Hospital Luis Calvo Mackenna, siguiendo recomendaciones para investigación en educación médica ${ }^{20,21}$.

\section{Resultados}

El carácter cualitativo de esta investigación permitió conocer las percepciones de los entrevistados en relación a las características ideales del docente de postgrado de pediatría de la Universidad de Chile. Se obtuvo 6 categorías que fueron características personales, actitudes del docente, formación docente, metodología docente, identificación con la institución y clima académico. Las categorías anteriores se desglosaron a su vez en subcategorías las cuales dan pie al siguiente listado de características ideales que se describe a continuación junto con una cita textual emitida por una autoridad (A), docente (D), APTEP (B), egresado (E) o director de departamento (DD). Cabe destacar, que desde el texto original se extrajeron las frases que mejor traducían la característica aludida, no solamente la que repetía literalmente la característica señalada sino que también la que se podía deducir del texto citado:

Comprometido. "...El tema del compromiso es esencial y como se nota el tema del compromiso, se nota en una persona que hace bien su trabajo..." (A).

Responsable. "...Bueno, creo que primero tiene que haber condiciones personales que se traduzcan en compromiso, responsabilidad y respeto por los valores..." (A).

Honesto. “...Una persona honesta, que fuera capaz de reflejar sus valores personales y servir de modelo para sus educandos..." (A).

Respetuoso. "...Otra característica es que el docente tiene que saber decir las cosas de forma respetuosa para que uno le entienda lo que quiere decir ..." (B).

Tolerante. “...Yo creo que es importante que los docentes sean tolerantes, tolerantes con la religión, con la vestimenta, con la opción sexual..." (B).

Acogedor. "...Que sea una persona acogedora, que acepte las dudas de uno y que no te haga sentir mal y que te ayude también..." (E).

Empático. “...Lo otro es quizás tener empatía, yo creo esa es otra característica que debiera tener un buen docente con la gente que uno está interactuando, empatía, por un lado, para que a uno lo sientan cercano para que tengan la confianza de discutir temas..." (D).

Prudente. "...En términos humanos, yo creo que tiene que ser una persona que transmita como acercarse a un niño de la manera más adecuada, que eso es algo que lo necesita un pediatra y que otra especialidad no necesita. O sea, que transmita el cómo dar confianza, cómo abordar al niño sin que se asuste..." (E).

Asertivo. "...Que sea asertivo en sus decisiones, que permita resolver los problemas mirándolos desde distintos puntos de vista..." (E). 
Líder. “...Además debería ser una persona capaz de formar equipo y con características de líder..." (A).

Con capacidades de autoevaluación y autorreflexión. "...Capacidad de autoevaluación que yo lo incorporo en los términos éticos, me parece fundamental, el poder decir como lo estoy haciendo en este proceso. La reflexión del proceso es fundamental..." (A).

Facilitador del aprendizaje. “...Creo que es muy importante que tú sirvas como guía, como un conductor en el proceso de enseñanza-aprendizaje..." (A).

Modelo a seguir. "...Que trabaje, que predique con el ejemplo, en el fondo. Que no sólo con su decir puedas aprender, si no que con el actuar y con el ver que es lo que hace. O sea, una persona que esté contigo y que tú puedas en el fondo copiar. Un modelo, por ejemplo..." (E).

Motivador. “...El docente tiene que ser motivador, absolutamente motivador, pero esa capacidad de motivar tiene que estar basado en algunas herramientas pedagógicas, nosotros tenemos que tener el aprendizaje de algunas técnicas, no basta con ser simpático o empático para llegar al alumno y motivarlo..." (D).

Comunicador. "...El tema de la comunicación es tan importante que tal como lo hacen en otras profesiones, debería haber un apoyo para formar comunicacionalmente a los docentes. Para expresarse bien, para manejar el interés de las personas, de los auditorios..." (A).

Con vocación. “...Hay que tener de verdad vocación y entrega, entrega a nosotros, porque por mucho que tenga mucha capacidad y muchos cursos si no hay vocación ni entrega es mal valorado..." (B).

Exigente. “...Es una forma de exigencia en el fondo y ellos sepan que nosotros no queremos que sean simpáticos y no exigentes...” (B).

Accesible. "...Debiera tener la capacidad de ser accesible, de tener una buena llegada, que uno tenga la confianza de preguntar..." (B).

Formado en docencia. "...El ideal sería que uno pudiese formar a los docentes. O sea aparece la persona que supone que tiene las características como docente y le damos la posibilidad de que desarrolle estas destrezas, en el fondo lo capacitamos para ser docente. No es un docente así espontáneo, sino que la formamos..." (DD).

Actualizado. "...Otra obligación es mantenerse actualizado, que es algo que uno debería asumir como un item consciente más que una rutina de trabajo, en que tú vayas revisando lo último..." (A).

Experto en su disciplina. "...A mi me parece que un docente en su disciplina debe ser a nivel clínico y académico, una persona del más alto nivel..." (A).

Investigador. "...El docente académico que no investiga, no puede ejercer la docencia. Esto debiera ser vital hacia donde debe ir un académico hoy día o hacia donde uno debe llevar un grupo. La expertise es práctica e investigación...” (DD).

Planificador. "...Creo que hay que planificar las actividades, ya sea teóricas o prácticas y cumplir con todo eso. Porque no es posible que un docente improvise...” (D).

Evaluador. “...Es central que conozca las herramientas de evaluación, que pueden ser obviamente distintas según los objetivos de los aprendizajes de un programa..." (A).

Con tiempo protegido para la docencia. "...Que idealmente el docente debe tener un horario dedicado a la docencia y no a las actividades asistenciales..." (D).

Conocedor y utilizador de nuevas TICS. “...No me cabe ninguna duda que las TICS son esenciales. Son herramientas tan poderosas que tienen que estar incorporadas y que facilitan ciertamente, bien usadas, el proceso, la metodología y pueden facilitar la evaluación...." (A)

Identificado con la institución. “...Tiene que haber un sentido de pertenencia, tener la camiseta puesta. Reconocer que hay falencias y todo, pero estar siempre con la actitud positiva hacia la Universidad..." (A).

Comprometido con la institución. “...Yo creo que el compromiso con la Universidad tiene que ser total, yo creo que un proceso docente sin la convicción de los valores que encierra una universidad, como en este caso nuestra universidad, la Universidad de Chile, valores transversales para toda la sociedad, que van a edificar la sociedad. Si uno no está empapado 
de eso es muy dificil transmitir con convicción $y$ con firmeza conceptos que esta comunidad de educandos pueda sentir que realmente son propios de la Universidad, por eso que el compromiso tiene que ser total..." (A).

Propiciador de un clima que facilite el aprendizaje. “...Tú tienes que tratar de llevar este proceso de facilitación y de enseñanzaaprendizaje en un clima agradable..." (A).

Capacidad de trabajo en equipo. "...La capacidad de trabajo en equipo es un atributo de la mayor importancia..." (DD).

Capacidad de revisar continuamente el proceso docente. "...Tiene que ser una persona abierta, receptiva, con una actitud critica y en esta actitud critica involucro a la necesa- ria cuota de preguntas que suscita el proceso docente de manera de generar una actitud de permanente evaluación..." (A).

Con capacidad de mantener buenas relaciones interpersonales. "...Establecer buenas relaciones interpersonales, es decir, no hablar mal del colega, no descalificar al resto del equipo. Esto también es algo deseable en la interacción con los pares y con todo el equipo con el cual se trabaja..." (DD).

Para facilitar la interpretación de los datos se muestra la figura 1 que muestra estas características del docente agrupadas según su rol como persona, profesor y clínico según lo propuesto por Sutkin ${ }^{22}$.

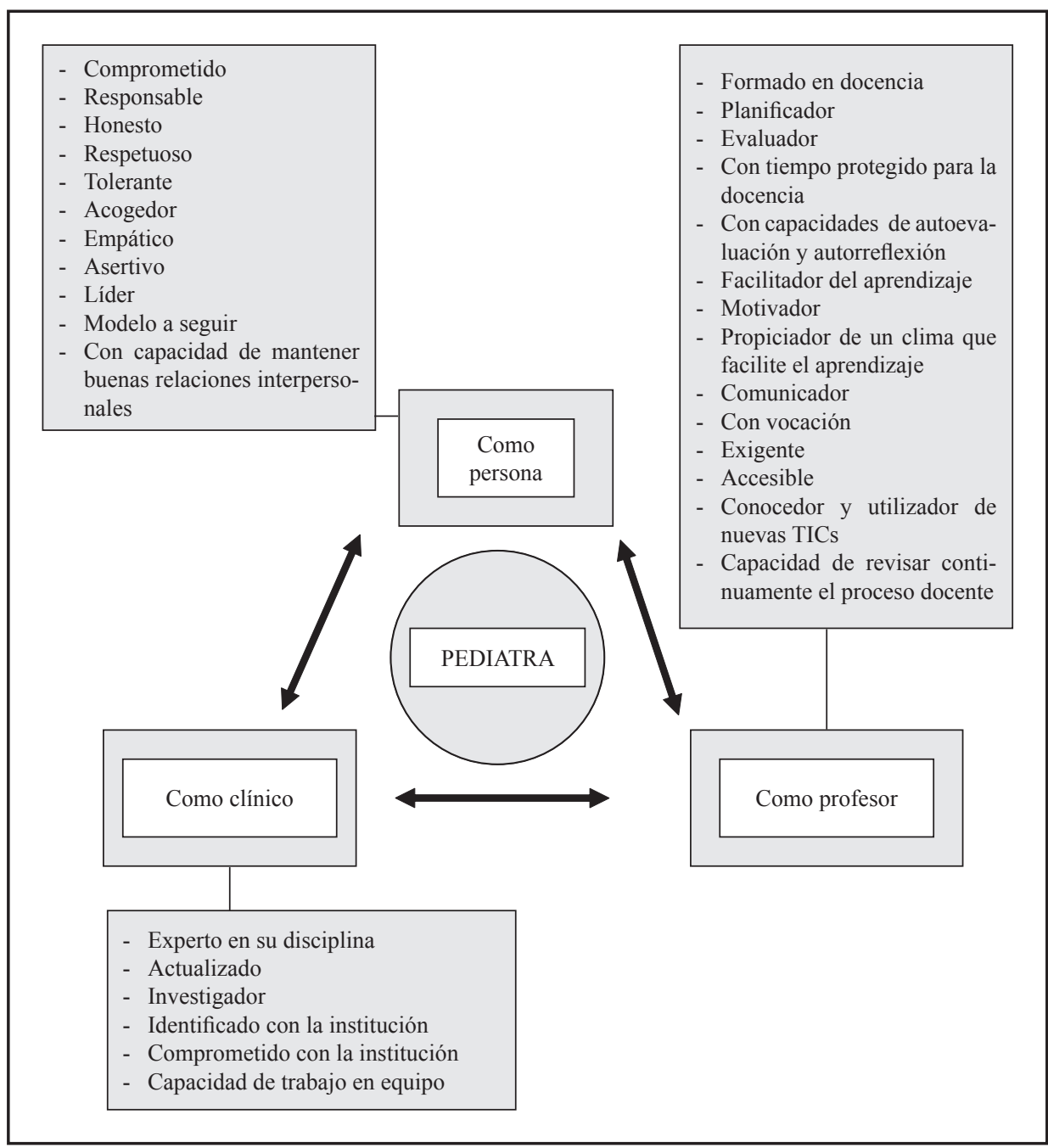

Figura 1. Esquema de características ideales del docente de postgrado de pediatría según rol como persona, docente y clínico. 


\section{Discusión}

Los documentos oficiales que describen características o competencias del docente de Postgrado de la Facultad de Medicina de la Universidad de Chile son escasos. A pesar de lo anterior, existen documentos que proponen algunas competencias genéricas con sello de la Universidad de Chile como responsabilidad social y compromiso ciudadano, capacidad crítica, capacidad autocrítica, compromiso ético, valoración y respeto por la diversidad y multiculturalidad, capacidad de trabajo en equipo, capacidad de expresión oral y escrita, habilidades en usar las tecnologías de la información y comunicación ${ }^{1}$.

Por su parte la Escuela de Postgrado de la Facultad de Medicina establece que sus docentes deben ser especialistas certificados o titulados por una universidad competente y deben poseer formación general y específica, experiencia, entrenamiento, calificación y certificación consistentes con los objetivos del programa en que se desempeñan ${ }^{2}$.

Es interesante analizar estas características definidas por documentos de la Universidad. Se destacan las semejanzas con lo encontrado en esta investigación en el sentido del deber ser experto en la disciplina, respetuoso, habituado en manejo de TICs, comunicador, capacidad autocrítica (o de autoevaluación y autorreflexión). Difieren otras características importantes que debe detentar cualquier médico como la capacidad crítica, compromiso ético, responsabilidad social y compromiso ciudadano y capacidad de trabajo en equipo.

En relación a lo anterior, es interesante contrastar nuestros resultados con la literatura. Para empezar, al señalar las competencias de un profesor modelo señaladas por Fielden $1998^{3}$, nos damos cuenta que nuestros hallazgos concuerdan por lo relatado por este experto en la Conferencia Mundial de Educación Superior al señalar como vitales el ser un experto en la disciplina, el conocer diversas estrategias de aprendizaje, metodología y de evaluación, conocimiento y aplicación de las nuevas tecnologías. Destaca aspectos no contemplados en nuestro trabajo como incorporar aspectos externos a la Universidad como son las influencias del mercado; además de tomar en cuenta la multiculturalidad como un aspecto que debe conocer y saber manejar. Estas diferencias podrían ser explicadas por el contexto internacional en el cual presenta estos resultados, pero es interesante señalar que uno de los hitos fundamentales al realizar las competencias del Proyecto Tuning fue solicitar información a empleadores y empresarios, entre otros $^{23}$.

En relación a un experto en el ámbito educativo como Miguel Zabalza, nuestra investigación concuerda con lo señalado en su libro ${ }^{4}$ en relación a ser un planificador del proceso, seleccionador de contenidos, conocer y realizar diferentes estrategias metodológicas y evaluativas, al igual que incorporar las nuevas tecnologías a la enseñanza. Este autor plantea y destaca otros elementos como lo son ser un creador de materiales de apoyo al aprendizaje, coordinar con colegas, revisar el proceso que no se encontraron en nuestro trabajo. Estos puntos son fundamentales porque nos hablan de capacidad didáctica, capacidad de trabajar en equipo y capacidad de feedback.

Con relación al docente médico Harden en su trabajo clásico ${ }^{5}$ define algunos roles que serían menester los tuviera los docentes médicos. En todo caso, este autor explora sólo la faceta del médico como profesor dejando de lado completamente las características personales y del clínico lo cual configura la principal diferencia con nuestra investigación. Ahora bien, en relación a la faceta como profesor se establecen las siguientes concordancias con nuestro trabajo a saber, el ser facilitador del aprendizaje, modelo a seguir, planificador, evaluador. Las diferencias cuando el autor señala el ser creador de recursos materiales y de guías de estudio, evaluador del currículum y conferencista.

Por su parte, Hesketh propone un marco de características de un docente médico basado en términos de resultados de aprendizaje del docente $^{6}$. Este trabajo se realizó sintetizando lo expuesto en la literatura por parte de un panel de expertos y el análisis de los currículos de diferentes programas de educación médica de Escocia. Siendo esta metodología la principal diferencia con nuestro trabajo. En todo caso, 
nuestra investigación concuerda con este autor en relación a que el docente debe saber de diversas estrategias metodológicas, de evaluación, planificación, de aprendizaje; además de tener formación docente y tener una serie de valores y actitudes como entusiasmo, empatía, respeto, etc. Este artículo explora sólo el área del docente como profesor y persona, dejando de lado las características técnicas que debe tener un docente clínico sí exploradas en nuestra investigación. Es interesante, además, que a pesar de lo extensa de estas competencias, no se señale la identificación y compromiso que deba existir con la institución universitaria que sí fue parte importante de nuestros resultados.

En relación al docente médico de pregrado, Martínez-González señala en su investigación realizada por un consenso de 35 expertos las competencias de este docente ${ }^{7}$. De esta manera, engloba 8 competencias subdivididas a su vez generando un total de 49 competencias. Estas competencias valoran el papel de facilitador del aprendizaje de los estudiantes logrando de esta manera guiar, entusiasmar y promover este proceso. Este trabajo no le da énfasis a las dimensiones del docente como clínico y en los aspectos del docente como persona lo engloba todo en su relación con el estudiante y tampoco se le da importancia al compromiso e identificación con la institución universitaria.

En relación al docente médico de postgrado, Martín Zurro en su calidad de experto propone una lista acotada de características ${ }^{8}$. Nuestra investigación concuerda con este autor en relación a ser experto en la disciplina, investigador y comunicador. Distinto a nuestro trabajo es cuando este autor señala que debería tener suficiente experiencia, referida a cantidad de años, este punto fue rechazado en el grupo focal de estudiantes en el cual señalan que un buen docente no es necesariamente quien tenga más años en el área.

Es interesante mencionar lo relatado por Sutkin en el año 2008 en que reportó en un artículo una revisión de la literatura analizando publicaciones desde el año 1909 hasta el 2006 acerca de las características que deben poseer los buenos docentes clínicos y se encontraron 480 características, englobadas en 49 temas respectivos y agrupados en 3 grandes catego- rías: docente como médico, docente como profesor y el docente como persona. Las principales características totales fueron: poseer conocimientos médicos y clínicos; poseer buenas herramientas clínicas y técnicas; competencia y buen razonamiento clínico; habilidad para favorecer relaciones positivas con los estudiantes y facilitar ambientes de aprendizaje de apoyo; poseer buenas herramientas de comunicación y entusiasmo ${ }^{22}$. En nuestra investigación encontramos las mismas características. Es destacable que nuestro trabajo ocupó una metodología cualitativa, en cambio, este autor también incorpora en su revisión la recopilación de ensayos y opiniones de expertos.

Es interesante señalar que la mayoría de las características determinadas por los entrevistados están en directa sintonía con lo expresado en términos del profesionalismo del pediatra descritos por el Comité de Bioética de la Academia Americana de Pediatría ${ }^{24}$, si bien es cierto, algunos valores fundamentales como el altruismo ${ }^{25}$ autosuperación, conocimiento de los propios límites, entre otros, no fueron expresados explícitamente por los entrevistados.

\section{Conclusiones}

En base a todo lo anterior, se puede concluir que la percepción del perfil de las características ideales del docente de postgrado de pediatría de la Facultad de Medicina de la Universidad de Chile podría resumirse de la siguiente manera: el docente ideal del postgrado de pediatría de la Facultad de Medicina de la Universidad de Chile es percibido por sus integrantes como un pediatra experto en la disciplina que enseña, identificado con la Universidad, con formación docente y con habilidades, actitudes, valores y conocimientos adecuados que faciliten el aprendizaje del estudiante todo dentro de un clima académico apropiado para este propósito.

\section{Referencias}

1.- http://www.lapetus.uchile.cl/lapetus/wordpress/recursos/programa_cfg_2009.html (última revisión el 07 de mayo de 2012). 
2.- Documento Estándares para los programas de título de especialistas. Escuela de Postgrado. Facultad de Medicina. Universidad de Chile. Mayo de 2007.

3.- Fielden J: (1998). Higher education staff development: continuing mission: thematic debate. World Conference on Higher Education. Higher Education in the Twentyfirst Century: Vision and Action. UNESCO. http:// unesdoc.unesco.org/images/0011/001173/117314e.pdf (última revisión el 07 de mayo de 2012).

4.- Zabalza MA: Competencias docentes del profesorado universitario. Calidad y desarrollo profesional. Madrid: Ediciones Narcea. 2003.

5.- Harden RM, Crosby JR: The good teacher is more than a lecturer: the twelve roles of the teacher. Medical Teacher 2000; 22 (4): 334-47.

6.- Hesketh E, Bagnall G, Buckley E, et al: A framework for developing excellence as a clinical educator. Medical Education 2001; 35: 555-64.

7.- Martinez-González A, López-Bárcena J, Herrera P, et al: Modelo de competencias del profesor de medicina. Educación Médica 2008; 11: 157-67.

8.- Martín Zurro A: Acreditación y reconocimiento de los tutores. Incentivación de la acción tutorial, Educación Médica 2003; 6 (3).

9.- Carr W: Capítulo II: Epistemología de la investigación científica. En: Hacia una ciencia crítica de la educación, Barcelona: Ediciones Laertes, 1990: 145-60.

10.- Bourdieu P: Espacio social y espacio simbólico. En Razones prácticas: sobre la teoría de la acción, Barcelona: Ed. Anagrama, 1997: 11-26.

11.- Habermas J: Conocimiento e interés. Madrid: Ediciones Taurus, 1982.

12.- Ruiz Olabuénaga J: Metodología de la investigación cualitativa. Bilbao: Universidad de Deusto, 2003.

13.- Quáas C: Paradigmas cuantitativos y cualitativos en la evaluación y seguimiento del profesional universitario. Revista Enfoques Educacionales 1998; 1 (1): 50-5.

14.- Mayorga M: La entrevista cualitativa como técnica de la evaluación de la docencia universitaria. Revista Electrónica de Investigación y Evaluación Educativa 2004; 10 (1): 23-39. http://www.uv.es/RELIEVE/v10n1/
RELIEVEv10n1_2.htm (última revisión el 07 de mayo de 2012).

15.- Osses $S$, Sánchez I, Ibáñez F: Investigación cualitativa en educación. Hacia la generación de teoría a través del proceso analítico. Estudios Pedagógicos (Valdivia) 2006; 32 (1): 119-33.

16.- Rodríguez G, Gil J, García E: Metodología de la investigación cualitativa. Málaga, España: Ediciones Aljibe, 1996.

17.- Sandín MP: Investigación cualitativa en educación. Fundamentos y tradiciones. Madrid: McGraw-Hill, 2003.

18.- Krueger RA: Focus groups: A practical guide for applied research. California: Beverly Hills, Sage, 1991.

19.- Muñoz Cantero JM, Ríos de Deus MP, Abalde E: (2002). Evaluación Docente vs Evaluación de la Calidad. Revista electrónica de investigación y evaluación educativa 8 (2). http://www.uv.es/RELIEVE/v8n2/ RELIEVEv8n2_4.htm. (última revisión el 07 de mayo de 2012).

20.- Morrison J, Prideaux D: Ethics approval for research in medical education. Medical Education 2001; 35: 1008.

21.- Pugsley L, Dornan T: Using a sledgehammer to crack a nut: clinical ethics review and medical education research projects. Medical Education 2007; 41: 726-8.

22.- Sutkin G, Wagner E, Harris I, Schiffer R: What makes a good clinical teacher in medicine?. A review of the literature. Academic Medicine 2008; 83: 452-66.

23.- Informe final-Proyecto Tuning América Latina. Reflexiones y perspectivas de la educación superior en América Latina (2007). Disponible en http:// tuning.unideusto.org/tuningal/index.php?option=com docman\&Itemid=191 (última revisión el 07 de mayo de 2012).

24.- Committee on Bioethics. American Academy of Pediatrics: Professionalism in Pediatrics: Statement of Principles. Pediatrics 2007; 120 (4): 895-7.

25.- Wojtczak A: (2002). Glossary of medical education terms. Disponible en http://www.iime.org/glossary.htm. (última revisión el 07 de mayo de 2012). 